\title{
Cinética de secagem e propriedades termodinâmicas da polpa de patauá (Oenocarpus bataua Mart.)
}

\section{Drying kinetics and thermodynamic properties of patawa pulp (Oenocarpus bataua Mart.)}

\section{Dyego da Costa Santos ${ }^{1 *}$ (D), Tiago Nascimento da Costa', Fabiana Braga Franco', Risle da Costa Castro1, João Paulo de Lima Ferreira², Marcel Alexandre da Silva Souza1, Johnson Clay Pereira Santos ${ }^{1}$}

${ }^{1}$ Instituto Federal de Educação, Ciência e Tecnologia do Acre (IFAC), Departamento de Tecnologia em Agroindústria, Xapuri/AC - Brasil

${ }^{2}$ Universidade Federal de Campina Grande (UFCG), Unidade Acadêmica de Engenharia Agrícola, Campina Grande/PB - Brasil

*Corresponding Author: Dyego da Costa Santos, Instituto Federal de Educação, Ciência e Tecnologia do Acre (IFAC), Departamento de Tecnologia em Agroindústria, Rua Coronel Brandão, 1622, Centro, CEP: 69930-000, Xapuri/AC - Brasil, e-mail: dyego.csantos@gmail.com

Cite as: Santos, D. C., Costa, T. N., Franco, F. B., Castro, R. C., Ferreira, J. P. L., Souza, M. A. S., \& Santos, J. C. P. (2019). Drying kinetics and thermodynamic properties of patawa pulp (Oenocarpus bataua Mart.). Brazilian Journal of Food Technology, 22, e2018305. https://doi.org/10.1590/1981-6723.30518

\begin{abstract}
Resumo
O patauá é uma fruta amazônica que, devido às suas propriedades sensoriais e nutricionais atrativas, tem despertado o interesse da comunidade científica. Entretanto, o elevado conteúdo de umidade confere alta perecibilidade ao produto fresco. Assim, objetivou-se estudar a cinética de secagem convectiva e determinar as propriedades termodinâmicas da polpa de patauá. A secagem foi realizada sob diferentes temperaturas $(40,50 \mathrm{e}$ $\left.60^{\circ} \mathrm{C}\right)$ e espessuras da camada $(0,3$ e $0,6 \mathrm{~cm})$, com velocidade do ar constante em $1,8 \mathrm{~m} \mathrm{~s}^{-1}$. Diferentes modelos de secagem em camada fina foram ajustados aos dados experimentais. $\mathrm{O}$ aumento da temperatura do ar e a diminuição da espessura da camada resultaram em aumento da taxa de remoção de umidade e, portanto, reduziram o tempo total de processo. Com base nos parâmetros estatísticos, o modelo de Midilli foi o que melhor descreveu a cinética de secagem das amostras para todas as condições avaliadas. Os coeficientes de difusão efetivos aumentaram com a elevação da temperatura do ar, apresentando magnitudes na ordem de $10^{-10} \mathrm{~m}^{2} \mathrm{~s}^{-1}$ e sua dependência com a temperatura foi representada pela equação de Arrhenius, com energia de ativação (Ea) de 32,41 e $40,10 \mathrm{~kJ} \mathrm{~mol}^{-1}$ para as espessuras de 0,6 e 0,3 cm, respectivamente. As variações de entalpia $(\Delta \mathrm{H})$ e de entropia $(\Delta \mathrm{S})$ reduziram-se com a elevação da temperatura de secagem, sendo menores na amostra desidratada na camada de $0,6 \mathrm{~cm}$, enquanto que a variação na energia livre de Gibbs $(\Delta G)$ foi aumentada com a ampliação da temperatura, sendo mais elevada na secagem com camada de $0,3 \mathrm{~cm}$.
\end{abstract}

Palavras-chave: Fruta amazônica; Desidratação; Modelagem matemática; Difusividade; Energia de ativação; Entalpia. 


\begin{abstract}
The patawa is an Amazonian fruit that, due to its sensory and nutritional properties, has aroused the interest of the scientific community. However, its high moisture content confers high perishability to the fresh product. Thus the convective drying kinetics were studied and the thermodynamic properties of the patawa pulp determined. Drying was carried out at different temperatures $\left(40,50\right.$ and $\left.60^{\circ} \mathrm{C}\right)$ and layer thicknesses $(0.3$ and $0.6 \mathrm{~cm})$, with a constant air velocity of $1.8 \mathrm{~m} \mathrm{~s}^{-1}$. Different thin layer drying models were adjusted to the experimental data. Increasing the air temperature and decreasing the layer thickness increased the moisture removal rate and thus reduced the overall process time. Based on the statistical parameters, the Midilli model was the one that best described the drying kinetics of the samples under all conditions evaluated. The effective diffusion coefficients increased with increase in air temperature, showing magnitudes to the order of $10^{-10} \mathrm{~m}^{2} \mathrm{~s}^{-1}$, and its temperature dependence was represented by the Arrhenius equation, with the activation energy (Ea) from 32.41 and $40.10 \mathrm{~kJ} \mathrm{~mol}^{-1}$ for the thicknesses of 0.6 and $0.3 \mathrm{~cm}$, respectively. The variations in enthalpy $(\Delta \mathrm{H})$ and entropy $(\Delta \mathrm{S})$ decreased with increasing drying temperature, being lowest in the sample dehydrated in a $0.6 \mathrm{~cm}$ layer, while the variation in Gibbs free energy $(\Delta G)$ increased with the increase in temperature, being higher when dried in a $0.3 \mathrm{~cm}$ layer.
\end{abstract}

Keywords: Amazonian fruit; Dehydration; Mathematical modelling; Diffusivity; Activation energy; Enthalpy.

\title{
1 Introdução
}

O patauazeiro (Oenocarpus bataua Mart.), da família Arecaceae, é uma palmeira nativa e típica da região Amazônica (Vieira et al., 2015), considerada uma espécie "oligárquica", cuja população natural produz cerca de 11 toneladas de frutos/ha/ano (Hidalgo et al., 2016). Seu fruto, popularmente conhecido no Brasil como patauá, é pequeno, com formato redondo, anelado e, quando está maduro, apresenta cor púrpura escura, costumando ser recoberto por uma camada cerosa e esbranquiçada (Vieira et al., 2015). A polpa é cerca de $40 \%$ do peso do fruto (Darnet et al., 2011), constituindo-se em fonte de proteínas, gorduras e carboidratos comparável à carne e ao leite materno (Guarín \& Valle, 2014), sendo também reportada como fonte de compostos bioativos, a exemplo de compostos fenólicos, que apresentam elevada atividade antioxidante (Rezaire et al., 2014). Rodrigues et al. (2010) relataram que a polpa de patauá apresenta $41,8 \%$ de gordura, em que a proporção de ácidos graxos insaturados é semelhante à do azeite de oliva, sendo inclusive consumida por populações locais.

O patauá constitui-se em uma alternativa de exploração sustentável e, por ser um material de características sensoriais inéditas no mercado, tem grande potencial como matéria-prima para a agroindústria. No entanto, o elevado teor de umidade do patauá, cerca de $40,76 \%$ b.u. (68\% b.s.) (Vieira et al., 2015), confere alta perecibilidade ao produto fresco, o que resulta em perdas pós-colheita em toda a cadeia produtiva, limitando o seu potencial de comercialização. Assim, para viabilizar a sua utilização em mercados distantes dos locais de ocorrência da espécie, deve-se considerar a utilização de tecnologias que possibilitem a extensão da vida útil do fruto. Nesse sentido, a secagem convectiva, por sua simplicidade e custo mais acessível em comparação a outros métodos, é uma das tecnologias mais utilizadas para a conservação de produtos agrícolas e oferece como vantagens o aumento da sua vida de prateleira, a minimização das perdas na pós-colheita do excesso da produção e, devido à contração volumétrica, a redução dos custos de embalagem, transportes e armazenamento (Santos et al., 2013; Corrêa-Filho et al., 2015). Os frutos desidratados poderão ser utilizados em processos de separação de seus constituintes para usos industriais diversos ou triturados para obtenção de um pó que poderá ser incorporado em formulações de alimentos.

Diversos estudos de secagem de frutos estão disponíveis na literatura nacional e internacional, e são realizados para avaliar o impacto da temperatura, espessura e/ou velocidade do ar de secagem nas características físicas do processo de desidratação (Wang et al., 2018; Silva et al., 2009, 2018; Sehrawat et al., 2018; Resende et al., 2018; Araújo et al., 2017a, 2017b; Feitosa et al., 2017; Alves \& 
Rodovalho, 2016). Apesar do interesse da comunidade acadêmica em relação ao tema, não foram encontradas pesquisas de secagem de patauá, o que reforça a necessidade de realização desta pesquisa.

Nesse sentido, objetivou-se estudar a cinética de secagem convectiva, nas temperaturas de 40,50 e $60{ }^{\circ} \mathrm{C}$, e espessuras de camada de 0,3 e $0,6 \mathrm{~cm}$, e determinar os coeficientes de difusão efetivos, a energia de ativação e as propriedades termodinâmicas do processo.

\section{Material e métodos}

Frutos de patauá (Oenocarpus bataua Mart.), safra 2018, em estádio de maturação maduros, observado pela coloração da casca púrpura escura, foram adquiridos em feira local da cidade de Xapuri, Acre, Brasil. Os frutos selecionados foram lavados com detergente neutro, sanitizados em solução clorada (100 ppm) por 15 min e enxaguados com água corrente para remoção do excesso de cloro. Posteriormente, procedeu-se à cocção da matéria-prima na temperatura de $50^{\circ} \mathrm{C}$ por $15 \mathrm{~min}$, com a finalidade de amaciar o mesocarpo. Após resfriamento, em temperatura ambiente $\left(25^{\circ} \mathrm{C}\right)$, a polpa do patauá (epicarpo e mesocarpo) foi separada manualmente do caroço e triturada em liquidificador industrial por $5 \mathrm{~min}$, até obtenção de uma polpa homogênea, que foi envasada em embalagens de polietileno de baixa densidade (PEBD) e armazenada em freezer a $-18{ }^{\circ} \mathrm{C}$ (Brasil, 1977).

Antes da secagem, a polpa foi descongelada sob refrigeração $\left(4{ }^{\circ} \mathrm{C}\right)$ por 24 horas e, em seguida, colocada em bancada de laboratório até que entrasse em equilíbrio térmico com o ambiente. Os experimentos foram conduzidos em triplicata, com três repetições, em desidratador de alimentos de bancada, modelo Pratic Drayer, nas temperaturas de 40,50 e $60{ }^{\circ} \mathrm{C}$, e espessuras de 0,3 e $0,6 \mathrm{~cm}$, com velocidade do ar constante em $1,8 \mathrm{~m} \mathrm{~s}^{-1}$, determinada por meio de um anemômetro digital (modelo ITTHAL-300, Instrutemp, Brasil). Cerca de $65 \mathrm{~g}$ e $120 \mathrm{~g}$ de polpa de patauá foram espalhados em bandejas de alumínio, formando amostras com espessuras de 0,3 e 0,6 cm, respectivamente, medidas com paquímetro digital (modelo Absolute, Mitutoyo, Brasil) com resolução de $0,01 \mathrm{~mm}$. As amostras foram desidratadas nas diferentes condições de secagem, monitorando-se a remoção da umidade por meio de pesagens, usando uma balança eletrônica (modelo AS5500C, Marte, Brasil) com precisão de 0,001 g, em intervalos de tempo regulares de 5, 10, 20, $30,60,120$ e $240 \mathrm{~min}$, até que as amostras atingissem massa constante. Os teores de umidade no início e no final das desidratações foram determinados gravimetricamente por secagem em estufa a $105{ }^{\circ} \mathrm{C}$ por $24 \mathrm{~h}$. A partir dos dados dos teores de umidade em cada tempo de secagem, calcularam-se os adimensionais de umidade, expressos pelas razões de umidade, de acordo com a Equação 1.

$R X=\frac{X-X_{e}}{X_{i}-X_{e}}$

em que: $\mathrm{RX}=$ adimensional de umidade (adimensional); $\mathrm{X}=$ teor de umidade, b.s.; $\mathrm{X}_{\mathrm{i}}=$ teor de umidade inicial, b.s.; $\mathrm{X}_{\mathrm{e}}=$ teor de umidade de equilíbrio, b.s.

Diferentes modelos matemáticos (Tabela 1), comumente utilizados na descrição da secagem de produtos agrícolas, foram ajustados aos dados experimentais da cinética de secagem da polpa de patauá, usando-se o software Statistica ${ }^{\circledR}$ versão 7.0, através de regressão não linear, pelo método Quasi-Newton (StatSoft, 2007).

Tabela 1. Modelos matemáticos ajustados às curvas de cinética de secagem da polpa de patauá.

\begin{tabular}{ccc}
\hline Designação & Modelo & Equação \\
\hline Dois termos & $\mathrm{RX}=\mathrm{a} \exp (-\mathrm{k} \mathrm{t})+\mathrm{b} \exp (-\mathrm{q} \mathrm{t})$ & $(2)$ \\
Logarítmico & $\mathrm{RX}=\mathrm{a} \exp (-\mathrm{k} \mathrm{t})+\mathrm{c}$ & $(3)$ \\
Midilli & $\mathrm{RX}=\mathrm{a} \exp \left(-\mathrm{k} \mathrm{t} \mathrm{t}^{\mathrm{n}}\right)+\mathrm{bt}$ & $(5)$ \\
Newton & $\mathrm{RX}=\exp (-\mathrm{k} \mathrm{t})$ & $(6)$ \\
Page & $\mathrm{RX}=\exp \left(-\mathrm{k} \mathrm{t} \mathrm{t}^{\mathrm{n}}\right.$ & \\
\hline
\end{tabular}

$\mathrm{RX}=$ adimensional de umidade da amostra, adimensional; $\mathrm{k}=$ constante de secagem; $\mathrm{a}, \mathrm{b}, \mathrm{c}, \mathrm{n}, \mathrm{q}=$ coeficientes dos modelos; $\mathrm{t}=$ tempo de secagem ( $\mathrm{min})$. 
Utilizaram-se, como critérios de ajuste das equações matemáticas aos dados de cinética de secagem da polpa de patauá, patauá, os valores dos coeficientes de determinação, bem como as magnitudes dos quiquadrados $(\chi 2)$ e dos desvios quadráticos médios (DQM) segundo as Equações 7 e 8, respectivamente, segundo as Equações 7 e 8 .

$$
\begin{aligned}
& \mathrm{DQM}=\left[\frac{1}{\mathrm{n}} \sum_{\mathrm{i}=1}^{\mathrm{n}}\left(\mathrm{RX}_{\mathrm{pred}, \mathrm{i}}-\mathrm{RX}_{\text {exp } \mathrm{i}}\right)^{2}\right]^{\frac{1}{2}} \\
& \chi^{2}=\frac{1}{\mathrm{n}-\mathrm{N}} \sum_{\mathrm{i}=1}^{\mathrm{n}}\left(\mathrm{RX}_{\text {exp }, \mathrm{i}}-\mathrm{RX}_{\text {pred, } \mathrm{i}}\right)^{2}
\end{aligned}
$$

em que: $\mathrm{RX}_{\exp }=$ adimensional de umidade experimental; $\mathrm{RX}_{\text {pred }}=$ adimensional de umidade predita pelo modelo; $\mathrm{N}$ = número de observações; $\mathrm{n}=$ número de constantes do modelo.

Os coeficientes de difusão efetivos foram determinados ajustando-se o modelo matemático da difusão líquida com aproximação de quatro termos (Equação 9) aos dados experimentais da cinética de secagem, considerando-se distribuição da umidade inicial uniforme, difusividade constante e resistência externa e contração do volume insignificantes. Este modelo é a solução analítica da segunda lei de Fick considerandose a forma geométrica das polpas como aproximada de uma placa plana (área >>espessura) (Crank, 1975).

$\mathrm{RX}=\frac{\mathrm{X}-\mathrm{X}_{\mathrm{e}}}{\mathrm{X}_{\mathrm{I}}-\mathrm{X}_{\mathrm{e}}}=\frac{8}{\pi^{2}} \sum_{\mathrm{n}=0}^{\infty} \frac{1}{(2 \mathrm{n}+1)^{2}} \exp \left[-(2 \mathrm{n}+1)^{2} \pi^{2}\right.$ Deff $\left.\frac{\mathrm{t}}{4 \mathrm{~L}^{2}}\right]$

em que: Deff $=$ coeficiente de difusão efetivo, $\mathrm{m}^{2} \mathrm{~s}^{-1} ; \mathrm{n}=$ número de termos da equação; $\mathrm{L}=$ dimensão característica (meia espessura da amostra), $\mathrm{m} ; \mathrm{t}=$ tempo, $\mathrm{s}$.

A relação entre os coeficientes de difusão efetivos e as temperaturas de secagem da polpa de patauá foi determinada, em cada espessura, com uso de uma equação do tipo Arrhenius (Equação 10).

$$
\text { Deff }=D_{0} \exp \left(-\frac{E_{a}}{R(T+273,15)}\right)
$$

em que: $\mathrm{D}_{\mathrm{o}}=$ fator pré-exponencial, $\mathrm{m}^{2} \mathrm{~s}^{-1} ; \mathrm{E}_{\mathrm{a}}=$ energia de ativação, $\mathrm{kJ} \mathrm{mol}^{-1} ; \mathrm{R}=$ constante universal dos gases, $0,008314 \mathrm{~kJ} \mathrm{~mol}^{-1} \mathrm{~K}^{-1} ; \mathrm{T}=$ temperatura, ${ }^{\circ} \mathrm{C}$.

A Equação 10 foi linearizada com aplicação de logaritmo natural para a obtenção dos parâmetros da equação de Arrhenius (Equação 11).

$\mathrm{LnD}=\mathrm{LnD}_{\mathrm{o}}-\frac{\mathrm{E}_{\mathrm{a}}}{\mathrm{R}} \cdot \frac{1}{\mathrm{~T}+273,15}$

em que: $\mathrm{LnD}_{\mathrm{o}}=$ logarítmico do fator pré-exponencial, $\mathrm{m}^{2} \mathrm{~s}^{-1} ; \mathrm{E}_{\mathrm{a}}=$ energia de ativação, $\mathrm{kJ} \mathrm{mol}^{-1} ; \mathrm{R}=$ constante universal dos gases, $0,008314 \mathrm{~kJ} \mathrm{~mol}^{-1} \mathrm{~K}^{-1} ; \mathrm{T}=$ temperatura, ${ }^{\circ} \mathrm{C}$.

As propriedades termodinâmicas de entalpia (Equação 12), entropia (Equação 13) e energia livre de Gibbs (Equação 14) do processo de secagem da polpa de patauá em diferentes temperaturas $\left(40,50\right.$ e $\left.60{ }^{\circ} \mathrm{C}\right)$ e espessuras $(0,3$ e $0,6 \mathrm{~cm})$ foram quantificadas por meio do método descrito por Silva et al. (2016).

$$
\begin{aligned}
& \Delta \mathrm{H}=\mathrm{E}_{\mathrm{a}}-\mathrm{R}(\mathrm{T}+273,15) \\
& \Delta \mathrm{S}=\mathrm{R}\left[\ln \left(\mathrm{D}_{\mathrm{o}}\right)-\ln \left(\frac{\mathrm{k}_{\mathrm{B}}}{\mathrm{h}_{\mathrm{p}}}\right)-\ln (\mathrm{T}+273,15)\right] \\
& \Delta \mathrm{G}=\Delta \mathrm{H}-(\mathrm{T}+273,15) \Delta \mathrm{S}
\end{aligned}
$$

em que: $\Delta \mathrm{H}=$ entalpia específica, $\mathrm{J} \mathrm{mol}^{-1} ; \Delta \mathrm{S}=$ entropia específica, $\mathrm{J} \mathrm{mol}^{-1} \mathrm{~K}^{-1} ; \Delta \mathrm{G}=$ energia livre de Gibbs, $\mathrm{J} \mathrm{mol}^{-1} ; \mathrm{K}_{\mathrm{B}}=$ constante de Boltzmann, $1,38 \times 10^{-23} \mathrm{~J} \mathrm{~K}^{-1} ; \mathrm{h}_{\mathrm{p}}=$ constante de Planck, $6,626 \times 10^{-34} \mathrm{~J} \mathrm{~s}^{-1}$; $\mathrm{T}=$ temperatura, ${ }^{\circ} \mathrm{C}$. 


\section{Resultados e discussão}

Nas curvas de secagem da polpa de patauá (Figura 1), que descrevem a evolução do teor de umidade em relação ao tempo de secagem, observa-se que o aumento da temperatura e a diminuição da espessura da camada diminuiu o tempo necessário para que as amostras atingissem o teor de umidade de equilíbrio. Os tempos de secagem, na espessura de $0,3 \mathrm{~cm}$, foram de 540,360 e 270 min nas temperaturas de 40,50 e $60^{\circ} \mathrm{C}$, respectivamente. Já na espessura de $0,6 \mathrm{~cm}$, foram de $1.200,720$ e 540 min respectivamente nas temperaturas de 40,50 e $60{ }^{\circ} \mathrm{C}$. O incremento na temperatura e a diminuição da espessura resultam no aumento da taxa de transferência de calor para o interior da espuma (Çengel \& Ghajar, 2012), provocando o aumento da taxa de remoção de umidade (Herman et al., 2018; Silva et al., 2018). Além disso, a redução da espessura diminui a distância que a água tem de percorrer do interior até a superfície da amostra (Silva et al., 2009; Djaeni et al., 2015), facilitando, assim, a sua remoção e, por consequência, reduzindo o tempo de secagem, como era esperado. Resultados similares foram relatados na literatura para a secagem de espuma de polpa de melão (Asokapandian et al., 2016), fatias de cenoura (Doymaz, 2017) e polpa de murta (Feitosa et al., 2017). Ainda que o aumento de temperatura reduza o tempo de secagem, sabe-se que tanto uma condição térmica elevada quanto a exposição excessiva ao calor podem degradar componentes termossensíveis. Logo, novas investigações devem ser realizadas para estudar a retenção de compostos bioativos e a manutenção da qualidade de nutrientes, como o óleo, a fim de estabelecer a melhor condição de secagem.

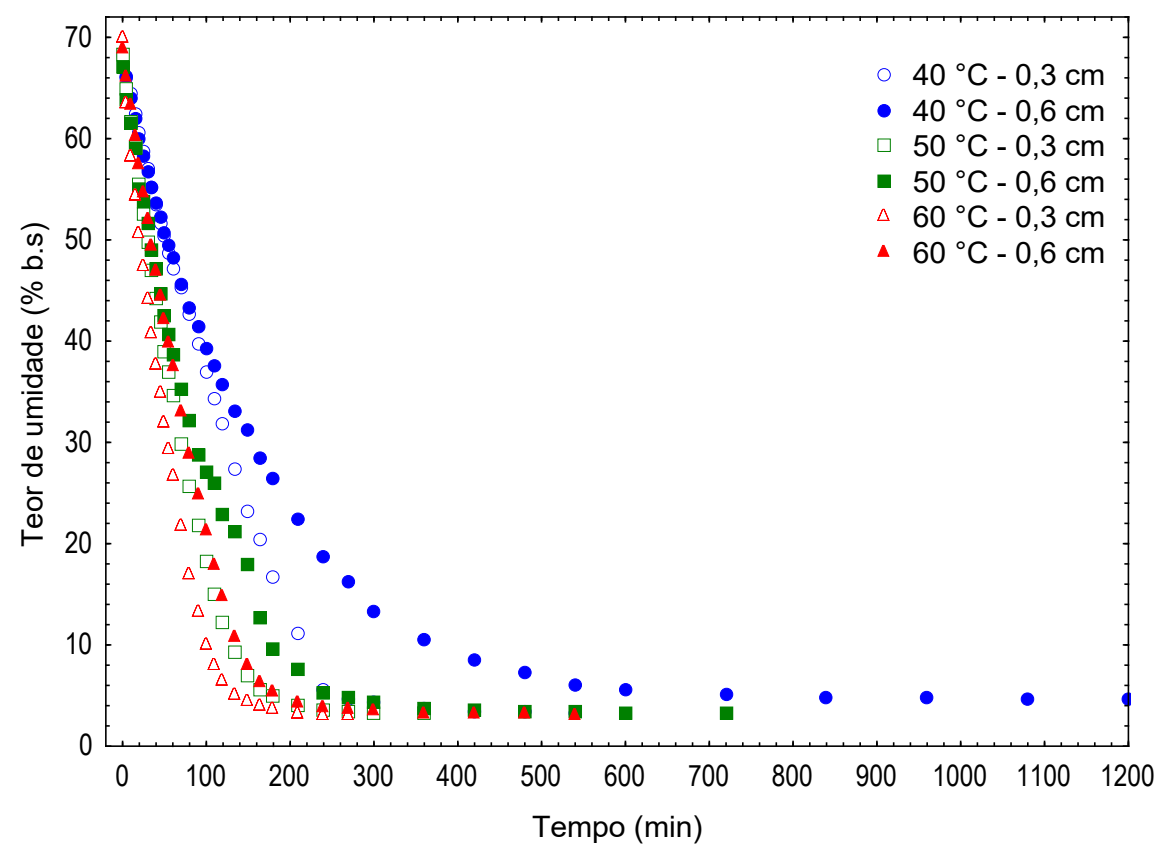

Figura 1. Evolução temporal do teor médio de umidade da polpa de patauá nas temperaturas de 40,50 e $60{ }^{\circ} \mathrm{C}$, e espessuras de 0,3 e $0,6 \mathrm{~cm}$.

Os parâmetros dos modelos matemáticos (Tabela 2) ajustados aos dados experimentais das curvas de cinética de secagem das amostras, em diferentes temperaturas e espessuras, demostram que o modelo de Midilli apresentou os maiores valores dos coeficientes de determinação $\left(\mathrm{R}^{2}>0,995\right)$ e os menores desvios quadráticos médios ( $\mathrm{DQM}<0,0179)$ e qui-quadrado $\left(\chi^{2}<5,9802\right)$, portanto, melhor representa o processo de secagem das amostras nas condições estudadas. 
Tabela 2. Parâmetros, coeficientes de determinação $\left(\mathrm{R}^{2}\right)$, desvios quadráticos médios (DQM) e qui-quadrados $\left(\chi^{2}\right)$ dos modelos matemáticos ajustados às curvas de cinética de secagem da polpa de patauá.

\begin{tabular}{|c|c|c|c|c|c|c|}
\hline Modelo & $\begin{array}{c}\begin{array}{c}\text { Espessura } \\
(\mathrm{cm})\end{array} \\
\end{array}$ & $\begin{array}{c}\text { Temperatura } \\
\left({ }^{\circ} \mathrm{C}\right) \\
\end{array}$ & Parâmetros do modelo & $\mathbf{R}^{2}$ & DQM & $\begin{array}{c}\chi^{2} \\
\left(\times 10^{-4}\right) \\
\end{array}$ \\
\hline \multirow{6}{*}{ 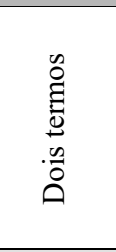 } & \multirow{3}{*}{0,3} & 40 & a: 0,$5264 ; \mathrm{k}: 0,0081 ; \mathrm{b}: 0,5265 ; \mathrm{q}: 0,0081$ & 0,9823 & 0,0454 & 23,7062 \\
\hline & & 50 & a: 0,$5311 ; \mathrm{k}: 0,0145 ; \mathrm{b}: 0,5299 ; \mathrm{q}: 0,0145$ & 0,9992 & 0,0094 & 0,9990 \\
\hline & & 60 & a: 0,$5138 ; \mathrm{k}: 0,0189 ; \mathrm{b}: 0,5137 ; \mathrm{q}: 0,0189$ & 0,9885 & 0,0359 & 15,0580 \\
\hline & \multirow{3}{*}{0,6} & 40 & a: 0,$4565 ; \mathrm{k}: 0,0061 ; \mathrm{b}: 0,5321 ; \mathrm{q}: 0,0061$ & 0,9955 & 0,0224 & 5,7053 \\
\hline & & 50 & a: 0,$5070 ; \mathrm{k}: 0,0105 ; \mathrm{b}: 0,5133 ; \mathrm{q}: 0,0105$ & 0,9900 & 0,0318 & 11,9468 \\
\hline & & 60 & a: 0,$5341 ; \mathrm{k}: 0,0132 ; \mathrm{b}: 0,5342 ; \mathrm{q}: 0,0132$ & 0,9885 & 0,0370 & 15,7225 \\
\hline \multirow{6}{*}{ 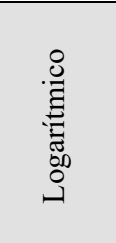 } & \multirow{3}{*}{0,3} & 40 & a: 1,$1274 ; \mathrm{k}: 0,0067 ; \mathrm{c}:-0,0927$ & 0,9889 & 0,0359 & 14,2922 \\
\hline & & 50 & a: 1,$1029 ; \mathrm{k}: 0,0128 ; \mathrm{c}:-0,0578$ & 0,9993 & 0,0086 & 0,7912 \\
\hline & & 60 & a: 1,$0618 ; \mathrm{k}: 0,0168 ; \mathrm{c}:-0,0500$ & 0,9927 & 0,0286 & 9,1599 \\
\hline & \multirow{3}{*}{0,6} & 40 & a: 0,$9953 ; \mathrm{k}: 0,0060 ; \mathrm{c}:-0,0090$ & 0,9964 & 0,0199 & 4,3609 \\
\hline & & 50 & a: 1,$0369 ; \mathrm{k}: 0,0099 ; \mathrm{c}:-0,0230$ & 0,9939 & 0,0249 & 7,0047 \\
\hline & & 60 & a: 1,$0955 ; \mathrm{k}: 0,0122 ; \mathrm{c}:-0,0368$ & 0,9908 & 0,0330 & 12,0355 \\
\hline \multirow{6}{*}{$\stackrel{7}{\Xi}$} & \multirow{3}{*}{0,3} & 40 & a: 0,$9559 ; \mathrm{k}: 0,0010 ; \mathrm{n}: 1,4051 ; \mathrm{b}:-3,8 \times 10^{-5}$ & 0,9955 & 0,0228 & 5,9802 \\
\hline & & 50 & a: 0,$9698 ; \mathrm{k}: 0,0030 ; \mathrm{n}: 1,3389 ; \mathrm{b}:-2,4 \times 10^{-5}$ & 0,9993 & 0,0089 & 0,8783 \\
\hline & & 60 & a: 0,$9542 ; \mathrm{k}: 0,0061 ; \mathrm{n}: 1,2533 ; \mathrm{b}:-4,9 \times 10^{-5}$ & 0,9987 & 0,0122 & 1,7467 \\
\hline & \multirow{3}{*}{0,6} & 40 & a: 0,$9830 ; \mathrm{k}: 0,0056 ; \mathrm{n}: 1,0161 ; \mathrm{b}:-0,6 \times 10^{-5}$ & 0,9974 & 0,0171 & 3,3295 \\
\hline & & 50 & a: 0,$9793 ; \mathrm{k}: 0,0054 ; \mathrm{n}: 1,1332 ; \mathrm{b}:-1,3 \times 10^{-5}$ & 0,9968 & 0,0179 & 3,7923 \\
\hline & & 60 & a: 0,$9703 ; \mathrm{k}: 0,0022 ; \mathrm{n}: 1,3800 ; \mathrm{b}:-0,4 \times 10^{-5}$ & 0,9986 & 0,0129 & 1,9212 \\
\hline \multirow{6}{*}{ 竞 } & \multirow{3}{*}{0,3} & 40 & $\mathrm{k}: 0,0075$ & 0,9779 & 0,0508 & 26,6688 \\
\hline & & 50 & $\mathrm{k}: 0,0135$ & 0,9990 & 0,0106 & 1,1464 \\
\hline & & 60 & $\mathrm{k}: 0,0183$ & 0,9840 & 0,0423 & 18,5843 \\
\hline & \multirow{3}{*}{0,6} & 40 & $\mathrm{k}: 0,0062$ & 0,9950 & 0,0236 & 5,7578 \\
\hline & & 50 & $\mathrm{k}: 0,0102$ & 0,9891 & 0,0332 & 11,4375 \\
\hline & & 60 & $\mathrm{k}: 0,0122$ & 0,9833 & 0,0445 & 20,4381 \\
\hline \multirow{6}{*}{\begin{tabular}{l}
$\infty$ \\
\multirow{2}{\infty}{} \\
$\approx$
\end{tabular}} & \multirow{3}{*}{0,3} & 40 & k: 0,$0020 ; \mathrm{n}: 1,2829$ & 0,9933 & 0,0280 & 8,3957 \\
\hline & & 50 & $\mathrm{k}: 0,0042 ; \mathrm{n}: 1,2734$ & 0,9991 & 0,0103 & 1,1278 \\
\hline & & 60 & k: 0,$0093 ; \mathrm{n}: 1,1695$ & 0,9979 & 0,0154 & 2,5425 \\
\hline & \multirow{3}{*}{0,6} & 40 & $\mathrm{k}: 0,0067 ; \mathrm{n}: 0,9866$ & 0,9970 & 0,0182 & 3,5436 \\
\hline & & 50 & k: 0,$0066 ; n: 1,2734$ & 0,9951 & 0,0222 & 5,3436 \\
\hline & & 60 & $\mathrm{k}: 0,0033 ; \mathrm{n}: 1,3033$ & 0,9980 & 0,0154 & 2,5239 \\
\hline
\end{tabular}

A relação entre os valores de adimensional de umidade experimentais e preditos pelo modelo de Midilli estão apresentados na Figura 2. Constata-se que os dados preditos se situaram próximos à linha reta que passa pela origem, o que representa similaridade entre os dados experimentais com aqueles preditos pelo modelo matemático (Alves \& Rodovalho, 2016). Isso se correlaciona positivamente com os parâmetros estatísticos $\mathrm{R}^{2}, \mathrm{DQM}$ e $\chi^{2}$, confirmando o ajuste adequado da equação de Midilli às curvas de cinética de secagem da polpa de patauá nas diferentes temperaturas e espessuras. Ao desidratarem a polpa de murta nas temperaturas de 50,60,70, 80 e $90^{\circ} \mathrm{C}$, e espessuras de 0,$5 ; 1,0$ e 1,5 cm, Feitosa et al. (2017) relataram que a equação de Midilli apresentou o melhor ajuste aos dados experimentais. Doymaz (2017) selecionou o modelo de Midilli para predizer o fenômeno de secagem de fatiais de cenoura com espessuras de 0,4;0,7 e 1,0 cm desidratadas nas temperaturas de 50,60 e $70{ }^{\circ} \mathrm{C}$. 


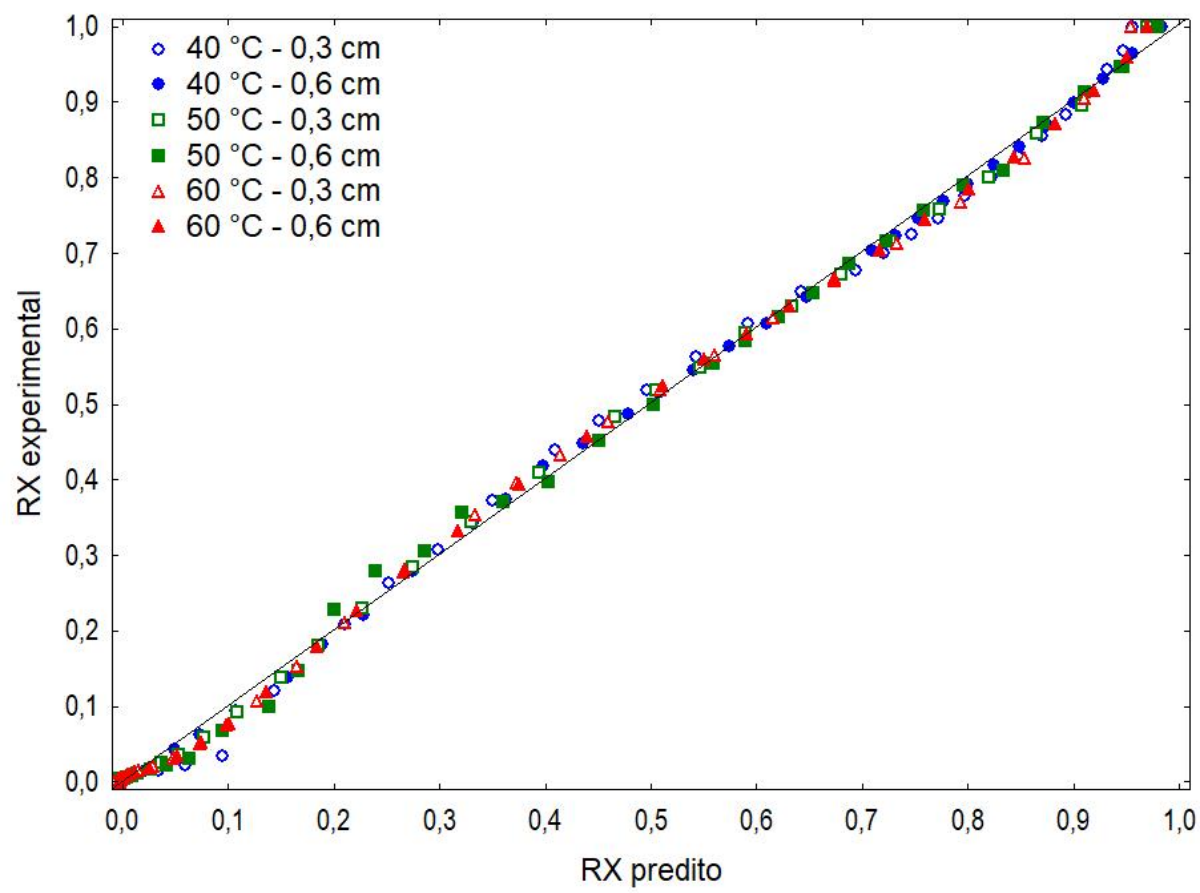

Figura 2. Relação entre os valores experimentais e preditos de adimensional do teor de umidade obtidos pelo modelo de Midilli na secagem da polpa de patauá nas temperaturas de 40,50 e $60^{\circ} \mathrm{C}$, e espessuras de 0,3 e $0,6 \mathrm{~cm}$.

A mudança média dos valores dos coeficientes de difusividade efetivos (Deff) com a temperatura e a espessura está apresentada na Tabela 3. De acordo com Cuevas et al. (2019), a difusividade efetiva de umidade (Deff) é um importante parâmetro de secagem para gêneros alimentícios, uma vez que é útil para projetar e modelar a transferência de massa durante este período, podendo variar com a espessura do material e as condições externas de secagem. Observa-se que os valores dos coeficientes de difusão variaram entre 0,5666 e $1,4245 \times 10^{-10} \mathrm{~m}^{2} \mathrm{~s}^{-1}$ na amostra com espessura de $0,3 \mathrm{~cm}$, e entre 2,6943 e $5,6702 \times 10^{-10} \mathrm{~m}^{2} \mathrm{~s}^{-1} \mathrm{na}$ espessura de 0,6 cm, apresentando-se na faixa relatada por Madamba et al. (1996) para gêneros alimentícios $\left(10^{-11}\right.$ a $\left.10^{-9} \mathrm{~m}^{2} \mathrm{~s}^{-1}\right)$. Resende et al. (2018) e Araújo et al. (2017b) também reportaram coeficientes de difusão de umidade na ordem de $10^{-10} \mathrm{~m}^{2} \mathrm{~s}^{-1} \mathrm{em}$ frutos de baru e de amendoim, respectivamente.

Tabela 3. Coeficientes de difusão efetivos $\left(\mathrm{m}^{2} \mathrm{~s}^{-1}\right)$ médios obtidos na secagem da polpa de patauá nas temperaturas de 40,50 e $60^{\circ} \mathrm{C}$, e espessuras de 0,3 e $0,6 \mathrm{~cm}$.

\begin{tabular}{cccc}
\hline Temperatura $\left({ }^{\circ} \mathbf{C}\right)$ & Espessura $(\mathbf{c m})$ & Deff $\left(\mathbf{m}^{\mathbf{2}} \mathbf{s}^{-1}\right)$ & $\mathbf{R}^{\mathbf{2}}$ \\
\hline \multirow{2}{*}{$40{ }^{\circ} \mathrm{C}$} & 0,3 & $0,5666 \times 10^{-10}$ & 0,9967 \\
& 0,6 & $2,6943 \times 10^{-10}$ & 0,9981 \\
\hline \multirow{2}{*}{$50^{\circ} \mathrm{C}$} & 0,3 & $1,0499 \times 10^{-10}$ & 0,9971 \\
& 0,6 & $4,6079 \times 10^{-10}$ & 0,9990 \\
\hline \multirow{2}{*}{$6{ }^{\circ} \mathrm{C}$} & 0,3 & $1,4245 \times 10^{-10}$ & 0,9962 \\
& 0,6 & $5,6702 \times 10^{-10}$ & 0,9981 \\
\hline
\end{tabular}

$\mathrm{R}^{2}=$ coeficiente de determinação.

Observa-se, ainda na Tabela 3, uma tendência de aumento de Deff com o incremento na temperatura e na espessura da amostra. O aumento da temperatura provoca maior agitação das moléculas de água, o que diminui a sua resistência ao escoamento, facilitando a sua difusão até a superfície da amostra (Guimarães et al., 2018; Resende et al., 2018). Além disso, é interessante destacar que, em uma temperatura fixa, a difusividade de umidade foi mais baixa na amostra com menor espessura, podendo esta diminuição relacionar-se com mudanças na estrutura da amostra, reflexo da formação de uma camada densa e pouco permeável na sua superfície, que provoca o aumento da resistência à transferência de massa 
(Wang et al., 2018). Esse comportamento foi igualmente descrito por Gomes et al. (2018) em massa triturada de jambu e por Rasouli et al. (2011) em fatias de alho, cujos coeficientes de difusão aumentaram com o aumento da espessura da camada.

Os coeficientes de difusão de umidade linearizados foram plotados com o inverso da temperatura de secagem (Figura 3) e a sua dependência com a temperatura do ar de secagem foi representada satisfatoriamente por uma equação do tipo Ahrrenius, que apresentou valores de $R^{2}$ superiores a 0,96 e 0,97 para as espessuras de 0,3 e $0,6 \mathrm{~cm}$, respectivamente.

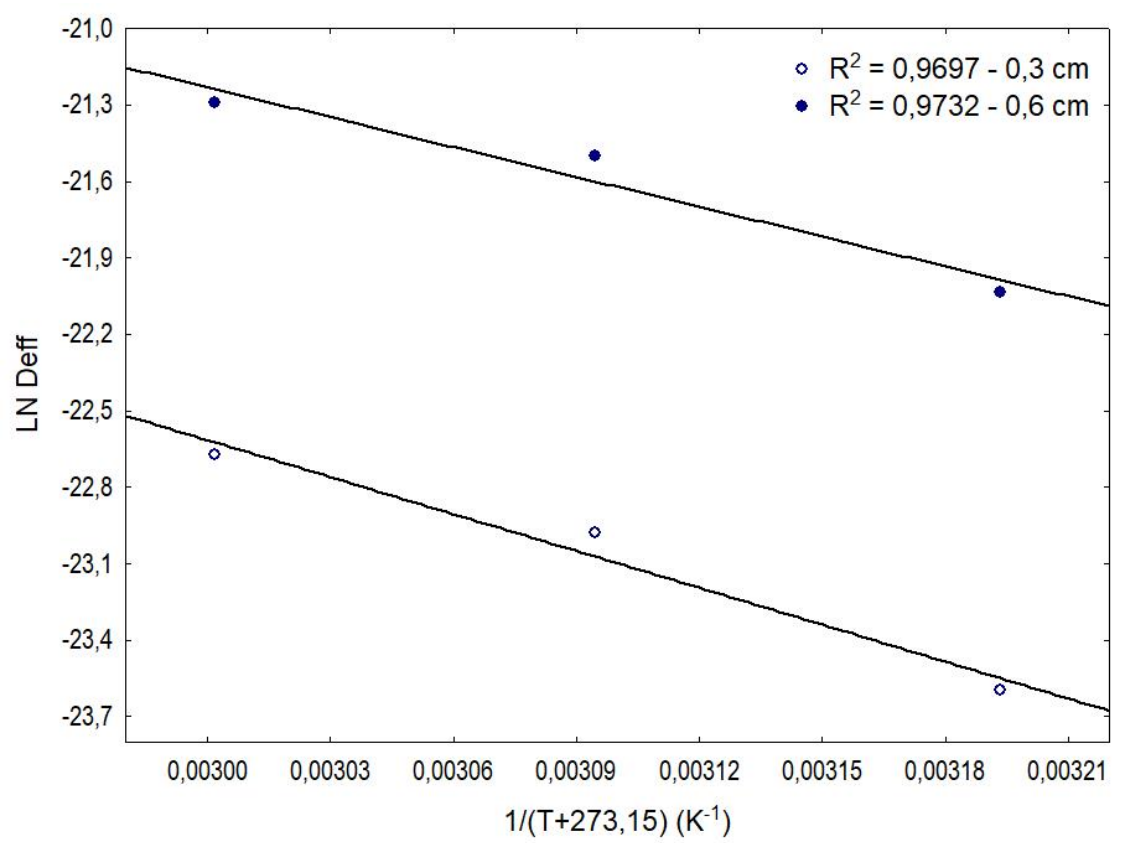

Figura 3. Representação de Arrhenius para os coeficientes de difusão efetivos médios obtidos na secagem da polpa de patauá nas temperaturas de 40,50 e $60{ }^{\circ} \mathrm{C}$, e espessuras de 0,3 a $0,6 \mathrm{~cm}$.

Da inclinação da curva da Equação do tipo Arrhenius, calcularam-se as energias de ativação da secagem da polpa de patauá em diferentes espessuras (Tabela 3). As Equações 15 e 16 apresentam os parâmetros da equação de Arrhenius ajustados aos coeficientes de difusão de umidade das polpas de patauá, para as espessuras de 0,3 e $0,6 \mathrm{~cm}$, respectivamente:

$\mathrm{D}_{\text {eff }}=2,9035 \times 10^{-4} \exp \left(-\frac{4823,71}{\mathrm{~T}+273,15}\right)$

$D_{\text {eff }}=7,2041 \times 10^{-4} \exp \left(-\frac{3897,77}{T+273,15}\right)$

A energia de ativação (Ea) para a secagem da polpa de patauá foi de $40,10 \mathrm{~kJ} \mathrm{~mol}^{-1}$ para a espessura de $0,3 \mathrm{~cm}$ e de $32,41 \mathrm{~kJ} \mathrm{~mol}^{-1}$ para a camada de $0,6 \mathrm{~cm}$, apresentando-se dentro da faixa de variação para gêneros alimentícios, que é de 12,70 a $110 \mathrm{~kJ} \mathrm{~mol}^{-1}$ (Zogzas et al., 1996). Sehrawat et al. (2018) evidenciaram Ea de 29,05 $\mathrm{kJ} \mathrm{mol}^{-1} \mathrm{em}$ cubos de manga secos nas temperaturas de 60,70 e $80{ }^{\circ} \mathrm{C}$, enquanto que Doymaz (2017) relatou valores de Ea de 35,53; 37,75 e 43,42 $\mathrm{kJ} \mathrm{mol}^{-1} \mathrm{em}$ fatias de cenoura. $\mathrm{O}$ aumento da espessura de 0,3 a $0,6 \mathrm{~cm}$ promoveu decréscimo de $19,18 \%$ na Ea, indicando que houve maior taxa de remoção de umidade na amostra com maior camada, uma vez que, quanto menor for o valor de Ea, maior será a difusividade, devido ao aumento do estado de vibração das moléculas de água (Younis et al., 2018). Isso pode estar relacionado às mudanças estruturais da amostra durante as secagens, como endurecimento e enrijecimento, conforme já discutido. De acordo com Kashaninejad et al. (2007), a Ea é uma barreira que deve ser ultrapassada, para que o processo de difusão 
possa ser desencadeado no produto, constituindo-se na energia mínima necessária para que as moléculas de água iniciem o movimento de dentro para fora do produto (Corrêa et al., 2005).

$\mathrm{Na}$ Tabela 4, estão apresentadas as propriedades termodinâmicas médias da polpa de patauá seca em diferentes temperaturas e espessuras. Observa-se que o aumento da temperatura do ar de secagem promove a redução da entalpia $(\Delta \mathrm{H})$, indicando que, em condições de maior temperatura, há menor demanda de energia térmica para promover a secagem da polpa de patauá, considerando-se que a $\Delta \mathrm{H}$ está relacionada com a energia necessária para remover a água ligada à matéria seca durante o processo de secagem (Resende et al., 2018). Esses valores ficaram compreendidos entre 37,3345 e $37,5008 \mathrm{~kJ} \mathrm{~mol}^{-1}$ para a amostra com espessura de $0,3 \mathrm{~cm}$ e entre 29,6363 e $29,8025 \mathrm{~kJ} \mathrm{~mol}^{-1}$ na polpa com camada de 0,6 cm. Araújo et al. (2017b) e Guimarães et al. (2018), estudando a secagem de okara e frutos de amendoim, respectivamente, relataram redução de $\Delta \mathrm{H}$ à medida que a temperatura de desidratação foi aumentada.

Tabela 4. Propriedades termodinâmicas médias da polpa de patauá secada nas temperaturas de 40,50 e $60{ }^{\circ} \mathrm{C}$, e espessuras de 0,3 e $0,6 \mathrm{~cm}$.

\begin{tabular}{ccccc}
\hline $\begin{array}{c}\text { Espessura } \\
(\mathbf{c m})\end{array}$ & $\begin{array}{c}\text { Temp. } \\
\left({ }^{\mathbf{C}} \mathbf{C}\right)\end{array}$ & $\begin{array}{c}\Delta \mathbf{H} \\
\left(\mathbf{k J ~ m o l}^{-\mathbf{1}}\right)\end{array}$ & $\begin{array}{c}\mathbf{\Delta S} \\
\left(\mathbf{k J ~ m o l}^{-\mathbf{1}} \mathbf{K}^{-\mathbf{1}}\right)\end{array}$ & $\begin{array}{c}\Delta \mathbf{G} \\
\left(\mathbf{k J} \mathbf{~ m o l}^{\mathbf{1}}\right)\end{array}$ \\
\hline & 40 & 37,5008 & $-0,3130$ & 135,5253 \\
0,3 & 50 & 37,4177 & $-0,3133$ & 138,6569 \\
& 60 & 37,3345 & $-0,3135$ & 141,7911 \\
\hline & 40 & 29,8025 & $-0,3246$ & 131,4560 \\
0,6 & 50 & 29,7194 & $-0,3249$ & 134,7034 \\
& 60 & 29,6363 & $-0,3251$ & 137,9535 \\
\hline
\end{tabular}

A entropia $(\Delta S)$, que é uma propriedade relacionada ao grau de excitação e arranjo espacial das moléculas de água em relação ao produto (Silva et al., 2016), ficou compreendida entre $-0,3130$ e $-0,3135 \mathrm{~kJ} \mathrm{~mol}^{-1} \mathrm{~K}^{-1}$ para a polpa com camada de $0,3 \mathrm{~cm}$ e entre $-0,3246$ e $-0,3251 \mathrm{~kJ} \mathrm{~mol}^{-1} \mathrm{~K}^{-1}$ para a amostra com espessura de 0,6 cm, aproximando-se dos valores reportados por Corrêa et al. (2011) em espigas de milho desidratadas nas temperaturas de 45,55 e $65^{\circ} \mathrm{C}$, que foram de $-0,3181$ a $-0,3176 \mathrm{~kJ} \mathrm{~mol}^{-1} \mathrm{~K}^{-1}$. De acordo com Cagnin et al. (2017), o aumento da temperatura provoca uma diminuição na $\Delta \mathrm{S}$ da amostra devido à diminuição do teor de umidade durante a desidratação, causando também dificuldade no movimento das moléculas de água no produto.

A energia livre de Gibbs $(\Delta \mathrm{G})$ apresentou-se com valores absolutos positivos, evidenciando que a secagem é um processo não espontâneo, sendo, portanto, necessário fornecer energia térmica para que possa ocorrer (Alves \& Rodovalho, 2016; Silva et al., 2016). A polpa de patauá desidratada com espessura de $0,3 \mathrm{~cm}$ deteve $\Delta \mathrm{G}$ variando de $135,5253 \mathrm{a} 141,7911 \mathrm{~kJ} \mathrm{~mol}^{-1}$, enquanto que a amostra com camada de $0,6 \mathrm{~cm}$ apresentou uma variação de 131,4560 a $137,9535 \mathrm{~kJ} \mathrm{~mol}^{-1}$. Ao determinarem as propriedades termodinâmicas de fatias de alho secas na faixa de temperatura de $40-60^{\circ} \mathrm{C}$, Cagnin et al. (2017) encontraram $\Delta \mathrm{G}$ compreendida entre 100,4334 e $103,9610 \mathrm{~kJ} \mathrm{~mol}^{-1}$.

\section{Conclusão}

Os tempos de desidratação são reduzidos com aumentos da temperatura do ar de secagem e da espessura da amostra, com respectivos decréscimos dos teores de umidade de equilíbrio. Todos os modelos matemáticos apresentam ajustes adequados aos dados experimentais. Entretanto, o modelo de Midilli foi selecionado como o mais adequado para predizer este processo, que apresenta coeficientes de difusão efetivos de umidade na ordem de $10^{-10} \mathrm{~m}^{2} \mathrm{~s}^{-1}$ e energias de ativação de 40,10 e $32,41 \mathrm{~kJ} \mathrm{~mol}^{-1}$ para as espessuras de 0,3 e $0,6 \mathrm{~cm}$, respectivamente. As propriedades termodinâmicas são afetadas pela temperatura de secagem e espessura de camada, com reduções da entalpia e da entropia, e aumento da energia de Gibbs, indicando um processo endergônico não espontâneo. Reforça-se a necessidade de estudos complementares, que 
correlacionem a temperatura de secagem com a retenção de compostos bioativos e com a qualidade dos demais componentes químicos, a exemplo do óleo.

\section{Referências}

Alves, J. J. L., \& Rodovalho, R. S. (2016). Cinética de secagem em camada de espuma da polpa de abacate CV 'Quintal' (Persea Americana Mill). Revista Agrotecnologia, 7(1), 86-98. http://dx.doi.org/10.12971/2179-5959/agrotecnologia.v7n1p86-98

Araújo, C. S., Macedo, L. L., Vimercati, W. C., Saraiva, S. H., Oliveira, A. N., \& Teixeira, L. J. Q. (2017a). Cinética de secagem de acerola em leito de espuma e ajuste de modelos matemáticos. Brazilian Journal of Food Technology, 20(1), 1-9. http://dx.doi.org/10.1590/1981-6723.15216

Araújo, W. D., Goneli, A. L. D., Corrêa, P. C., Hartmann Filho, C. P., \& Martins, E. A. S. (2017b). Modelagem matemática da secagem dos frutos de amendoim em camada delgada. Ciência Agronômica, 48(3), 448-457.

Asokapandian, S., Venkatachalam, S., Swamy, G. J., \& Kuppusamy, K. (2016). Optimization of foaming properties and foam mat drying of muskmelon using soy protein. Journal of Food Process Engineering, 39(6), 692-701. http://dx.doi.org/10.1111/jpe.12261

Brasil. Ministério da Saúde. Agência Nacional de Vigilância Sanitária - ANVISA. (1977). Resolução CNNPA nº 35, de 27 de dezembro de 1977. Diário Oficial da União, Brasília.

Cagnin, C., Lima, M. S., Silva, R. M., Silva, M. A. P., Plácido, G. R., Freitas, B. S. M., \& Oliveira, D. E. C. (2017). Garlic: Kinetic drying and thermodynamic properties. Bioscience Journal, 33(4), 905-913. http://dx.doi.org/10.14393/BJ-v33n4a2017-36886

Çengel, Y. A., \& Ghajar, A. J. (2012). Transfer of mass and heat (4th ed.). New York: McGraw-Hill.

Corrêa, P. C., Botelho, F. M., Oliveira, G. H. H., Goneli, A. L. D., Resende, O., \& Campos, S. C. (2011). Mathematical modeling of the drying process of corn ears. Acta Scientiarum. Agronomy, 33(4), 575-581. $\mathrm{http} / / / \mathrm{dx}$.doi.org/10.4025/actasciagron.v33i4.7079

Corrêa, P. C., Goneli, A. L. D., Resende, O., \& Ribeiro, D. M. (2005). Obtenção e modelagem das isotermas de dessorção e do calor isostérico de dessorção para grãos de trigo. Revista Brasileira de Produtos Agroindustriais, 7(1), 39-48. http://dx.doi.org/10.15871/1517-8595/rbpa.v7n1p39-48

Corrêa-Filho, L. C., Andrade, E. T., Martinazzo, A. P., D’Andrea, E. M., Sousa, F. A., \& Figueira, V. G. (2015). Cinética de secagem, contração volumétrica e análise da difusão líquida do figo (Ficus carica L.). Revista Brasileira de Engenharia Agrícola e Ambiental, 19(8), 797-802. http://dx.doi.org/10.1590/1807-1929/agriambi.v19n8p797-802

Crank, J. (1975). Mathematics of diffusion (2nd ed.). Oxford: Oxford University Press.

Cuevas, M., Martínez-Cartas, M. L., Pérez-Villarejo, L., Hernández, L., García-Martín, J. F., \& Sánchez, S. (2019). Drying kinetics and effective water diffusivities in olive stone andolive-tree pruning. Renewable Energy, 132(1), 911-920. http://dx.doi.org/10.1016/j.renene.2018.08.053

Darnet, S. H., Silva, L. H. M., Rodrigues, A. M. C., \& Lins, R. T. (2011). Nutritional composition, fatty acid and tocopherol contents of buriti (Mauritia flexuosa) and patawa (Oenocarpus bataua) fruit pulp from the Amazon region. Food Science and Technology (Campinas), 31(2), 488-491. http://dx.doi.org/10.1590/S0101-20612011000200032

Djaeni, M., Prasetyaningrum, A., Sasongko, S. B., Widayat, W., \& Hii, C. L. (2015). Application of foam-mat drying with egg white for carrageenan: Drying rate and product quality aspects. Journal of Food Science and Technology, 52(2), 1170-1175. PMid:25694735. http://dx.doi.org/10.1007/s13197-013-1081-0

Doymaz, I. (2017). Drying kinetics, rehydration and colour characteristics of convective hot-air drying of carrot slices. Heat and Mass Transfer, 53(1), 25-35. http://dx.doi.org/10.1007/s00231-016-1791-8

Feitosa, R. M., Figueirêdo, R. M. F., Queiroz, A. J. M., Lima, F. C. S., \& Oliveira, E. N. A. (2017). Drying and characterization of myrtle pulp. Revista Brasileira de Engenharia Agrícola e Ambiental, 21(12), 858-864. http://dx.doi.org/10.1590/18071929/agriambi.v21n12p858-864

Gomes, F. P., Osvaldo, R., Sousa, E. P., Oliveira, D. E. C., \& Araújo Neto, F. R. (2018). Drying kinetics of crushed mass of 'jambu': Effective diffusivity and activation energy. Revista Brasileira de Engenharia Agrícola e Ambiental, 22(7), 499-505. http://dx.doi.org/10.1590/1807-1929/agriambi.v22n7p499-505

Guarín, J. R., \& Valle, J. I. (2014). Modeling the stipe growth of the oenocarpus bataua palm in the Central Cordillera of the Andes, Colombia. Forest Ecology and Management, 314(1), 141-149. http://dx.doi.org/10.1016/j.foreco.2013.12.005

Guimarães, R. M., Oliveira, D. E. C., Resende, O., Silva, J. S., Rezende, T. A. M., \& Egea, M. B. (2018). Thermodynamic properties and drying kinetics of 'okara'. Revista Brasileira de Engenharia Agrícola e Ambiental, 22(6), 418-423. http://dx.doi.org/10.1590/1807-1929/agriambi.v22n6p418-423

Herman, C., Spreutels, L., Turomzsa, N., Konagano, E. M., \& Haut, B. (2018). Convective drying of fermented Amazonian cocoa beans (Theobroma cacao var. Forasteiro): Experiments and mathematical modeling. Food and Bioproducts Processing, 108(1), 81-94. http://dx.doi.org/10.1016/j.fbp.2018.01.002

Hidalgo, P. S. P., Nunomura, R. C. S., \& Nunomura, S. M. (2016). Plantas oleaginosas amazônicas: Química e atividade antioxidante de patauá (Oenocarpus bataua Mart.). Revista Virtual de Química, 8(1), 130-140.

Kashaninejad, M., Mortazavi, A., Safekordi, A., \& Tabil, L. G. (2007). Thin-layer drying characteristics and modeling of pistachio nuts. Journal of Food Engineering, 78(1), 98-108. http://dx.doi.org/10.1016/j.jfoodeng.2005.09.007 
Madamba, P. S., Driscoll, R. H., \& Buckle, K. A. (1996). Thin layer drying characteristics of garlic slices. Journal of Food Engineering, 29(1), 75-97. http://dx.doi.org/10.1016/0260-8774(95)00062-3

Rasouli, M., Seiiedlou, S., Ghasemzadeh, H. R., \& Nalbandi, H. (2011). Influence of drying conditions on the effective moisture diffusivity and energy of activation during the hot air drying of garlic. Australian Journal of Agricultural Engineering, 2(4), 96-101.

Resende, O., Oliveira, D. E. C., Costa, L. M., \& Ferreira Júnior, W. N. (2018). Drying kinetics of baru fruits (Dipteryx alata Vogel). Engenharia Agrícola, 38(1), 103-109. http://dx.doi.org/10.1590/1809-4430-eng.agric.v38n1p103-109/2018

Rezaire, A., Robinson, J. C., Bereau, D., Verbaere, A., Sommerer, N., Khan, M. K., Durand, P., Prost, E., \& Fils-Lycaon, B. (2014). Amazonian palm Oenocarpus bataua ("patawa"): Chemical and biological antioxidant activity: Phytochemical composition. Food Chemistry, 149(1), 62-70. PMid:24295677. http://dx.doi.org/10.1016/j.foodchem.2013.10.077

Rodrigues, A. M. C., Darnet, S., \& Silva, L. H. M. (2010). Fatty acid profiles and tocopherol contents of buriti (Mauritia flexuosa), patawa (Oenocarpus bataua), tucuma (Astrocaryum vulgare), mari (Poraqueiba paraensis) and inaja (Maximiliana maripa) fruits. Journal of the Brazilian Chemical Society, 21(10), 2000-2004. http://dx.doi.org/10.1590/S0103-50532010001000028

Santos, D. C., Queiroz, A. J. M., Figueirêdo, R. M. F., \& Oliveira, E. N. A. (2013). Cinética de secagem de farinha de grãos residuais de urucum. Revista Brasileira de Engenharia Agrícola e Ambiental, 17(2), 223-231. http://dx.doi.org/10.1590/S141543662013000200014

Sehrawat, R., Nema, P. K., \& Kaur, B. P. (2018). Quality evaluation and drying characteristics of mango cubes dried using lowpressure superheated steam, vacuum and hot air drying methods. Lebensmittel-Wissenschaft + Technologie, 92(1), 548-555. http://dx.doi.org/10.1016/j.lwt.2018.03.012

Silva, A. S., Melo, K. S., Alves, N. M. C., \& Gomes, J. P. (2009). Cinética de secagem em camada fina da banana maçã em secador de leito fixo. Revista de Biologia e Ciências da Terra, 9(2), 107-115. http://dx.doi.org/10.15871/15178595/rbpa.v11n2p129-136

Silva, H. W., Rodovalho, R. S., Velasco, M. F., Silva, C. F., \& Vale, L. S. R. (2016). Kinetics and thermodynamic properties related to the drying of 'Cabacinha' pepper fruits. Revista Brasileira de Engenharia Agrícola e Ambiental, 20(2), 174-180. http://dx.doi.org/10.1590/1807-1929/agriambi.v20n2p174-180

Silva, H., Vale Junior, L. S. R., Silva, C. F., Souza, R. C., \& Soares, R. S. (2018). Drying kinetics and physiological quality of 'Cabacinha' pepper seeds during storage. Revista Brasileira de Engenharia Agrícola e Ambiental, 22(4), 292-297. http://dx.doi.org/10.1590/1807-1929/agriambi.v22n4p292-297

StatSoft. (2007). Statistica for Window Computer programa manual: Versão 7.0. Tulsa: Statsoft Inc.

Vieira, T. S., Seixas, F. R. F., \& Cintra, D. E. C. (2015). Caracterização físico-química e da fração lipídica do patauá proveniente da aldeia baixa verde no município de Alto Alegre dos Parecis-RO. Revista Científica da UNESC, 13(16), 95-103.

Wang, J., Law, C. L., Nema, P. K., Zhao, J. H., Liu, Z. L., Deng, L. Z., Gao, Z. J., \& Xiao, H. W. (2018). Pulsed vacuum drying enhances drying kinetics and quality of lemon slices. Journal of Food Engineering, 224(1), 129-138. http://dx.doi.org/10.1016/j.jfoodeng.2018.01.002

Younis, M., Abdelkarim, D., \& Zein El-Abdein, A. (2018). Kinetics and mathematical modeling of infrared thin-layer drying of garlic slices. Saudi Journal of Biological Sciences, 25(2), 332-338. PMid:29472787. http://dx.doi.org/10.1016/j.sjbs.2017.06.011

Zogzas, N. P., Maroulis, Z. B., \& Marinos-Kouris, D. (1996). Moisture diffusivity data compilation in foodstuffs. Drying Technology, 14(10), 2225-2253. http://dx.doi.org/10.1080/07373939608917205 